\title{
BMJ Open Chinese herbal medicine for diabetic kidney disease: a systematic review and meta-analysis of randomised placebo- controlled trials
}

\author{
La Zhang, ${ }^{\oplus 1,2}$ Lihong Yang, ${ }^{2,3}$ Johannah Shergis, ${ }^{4}$ Lei Zhang, ${ }^{1}$ Anthony Lin Zhang, ${ }^{4}$ \\ Xinfeng Guo, ${ }^{2,3}$ Xindong Qin, ${ }^{1}$ David Johnson, ${ }^{5,6}$ Xusheng Liu, ${ }^{1}$ Chuanjian Lu, ${ }^{2,7}$ \\ Charlie Changli Xue, ${ }^{4}$ Wei Mao ${ }^{1}$
}

To cite: Zhang L, Yang L, Shergis J, et al. Chinese herbal medicine for diabetic kidney disease: a systematic review and meta-analysis of randomised placebocontrolled trials. BMJ Open 2019;9:e025653. doi:10.1136/ bmjopen-2018-025653

- Prepublication history and additional material for this paper are available online. To view these files, please visit the journal online (http://dx.doi org/10.1136/bmjopen-2018025653).

\section{CCX and WM contributed} equally.

LaZ and LY contributed equally.

Received 31 July 2018

Revised 7 February 2019

Accepted 5 March 2019

Check for updates

(c) Author(s) (or their employer(s)) 2019. Re-use permitted under CC BY-NC. No commercial re-use. See rights and permissions. Published by BMJ.

For numbered affiliations see end of article.

Correspondence to Dr Charlie Changli Xue; charlie.xue@rmit.edu.au and Professor Wei Mao; maowei1274@126.com

\section{ABSTRACT}

Objectives To provide a broad evaluation of the efficacy and safety of oral Chinese herbal medicine (CHM) as an adjunctive treatment for diabetic kidney disease (DKD), including mortality, progression to end-stage kidney disease (ESKD), albuminuria, proteinuria and kidney function.

Design A systematic review and meta-analysis. Methods Randomised controlled trials (RCTs) comparing oral CHM with placebo as an additional intervention to conventional treatments were retrieved from five English (Cochrane Central Register of Controlled Trials, MEDLINE, Embase, Allied and Complementary Medicine Database and Cumulative Index of Nursing and Allied Health Literature) and four Chinese databases (China BioMedical Literature, China National Knowledge Infrastructure, Chonqing VIP and Wanfang) from inception to May 2018. RCTs recruiting adult DKD patients induced by primary diabetes were considered eligible, regardless of the form and ingredients of oral CHM. Mean difference (MD) or standardised mean difference (SMD) was used to analyse continuous variables and $\mathrm{RR}$ for dichotomous data. Results From 7255 reports retrieved, 20 eligible studies involving 2719 DKD patients were included. CHM was associated with greater reduction of albuminuria than placebo, regardless of whether renin-angiotensin system (RAS) inhibitors were concurrently administered (SMD -0.56, $95 \% \mathrm{Cl}[-1.04$ to -0.08$\left.], \mathrm{I}^{2}=64 \%, \mathrm{p}=0.002\right)$ or not (SMD $-0.92,95 \% \mathrm{Cl}[-1.35$ to -0.51$\left.], \mathrm{I}^{2}=87 \%, \mathrm{p}<0.0001\right)$. When CHM was used as an adjunct to RAS inhibitors, estimated glomerular filtration rate was higher in the CHM than placebo group (MD $6.28 \mathrm{~mL} / \mathrm{min} ; 95 \% \mathrm{Cl}$ [2.42 to 10.14$], \mathrm{I}^{2}=0 \%$, $\mathrm{p}=0.001$ ). The effects of CHM on progression to ESKD and mortality were uncertain due to low event rates. The reported adverse events in CHM group included digestive disorders, elevated liver enzyme level, infection, anaemia, hypertension and subarachnoid haemorrhage, but the report rates were low and similar to control groups. The favourable results of CHM should be balanced with the limitations of the included studies such as high heterogeneity, short follow-up periods, small numbers of clinical events and older patients with less advanced disease.

Conclusions Based on moderate to low quality evidence, CHM may have beneficial effects on renal function and albuminuria beyond that afforded by conventional
Strengths and limitations of this study

- This systematic review and meta-analysis provided a broad review of the efficacy and safety of oral Chinese herbal medicine (CHM) for diabetic kidney disease.

- Randomised controlled trials comparing CHM to placebo were included to avoid potential risk of bias that may exaggerate the estimated effect of CHM.

- The search strategy was comprehensive, over 7000 articles were screened and 20 studies included with a total of 2719 participants.

- A priori subgroups analysis was performed to provide potential candidate formulae and frequently used herbs for further investigation.

- Overall the evidence was moderate to very low quality due to unclear randomisation procedures, wide $\mathrm{C}$ and substantial heterogeneity in outcome measures. The external validity was compromised by multi-ingredients herbal formulae, short follow-up periods, small numbers of clinical events and inclusion of older patients with less advanced disease.

treatment in adults with DKD. Further well-conducted, adequately powered trials with representative DKD populations are warranted to confirm the long-term effect of CHM, particularly on clinically relevant outcomes. PROSPERO registration number CRD42015029293.

\section{INTRODUCTION}

Diabetic kidney disease (DKD) is one of the most common complications of diabetes. As the prevalence of diabetes continues to grow globally, it is estimated that the number of DKD patients will double by $2025 .{ }^{1}$ Since patients with DKD are at markedly higher risks of progression to end-stage kidney disease (ESKD) and cardiovascular disease, the socioeconomic and public health burden of DKD is significant. ${ }^{2}{ }^{3}$ Effective therapies that prevent and treat DKD are of critical importance. 
Glycaemic management, blood pressure control and the renin-angiotensin system (RAS) inhibitors are the mainstay of treatment for DKD and have been successful in reducing risk of disease onset or progression. ${ }^{4}{ }^{5}$ However, an unmet need exists in DKD patients intolerant or unresponsive to current pharmacotherapies and those patients with deteriorating renal function yet normo-albuminuria. ${ }^{6-8}$ Some promising therapies addressing novel targets, such as sulodexide and bardoxolone methyl, have been found to be ineffective and/or harmful, while several others, including sodium-glucose cotransporter 2 inhibitors and mineralocorticoid receptor antagonist are still under evaluation. ${ }^{9-11}$

To facilitate the discovery of new therapeutic agents for patients with diabetes and impaired renal function, screening candidates from natural products including Chinese herbal medicine (CHM) that have traditionally been used for symptoms associated with DKD, may offer insights into a more targeted approach for therapeutic development. With respect to CHM, records dating to the Han dynasty (AD 202-220) indicate the treatment of DKD symptoms in Chinese medicine literature and contemporary literature including RCTs indicating CHM is used for diabetes and its complications. ${ }^{12}$ Multi-ingredient herbal decoctions and manufactured products of Abelmoschi Corolla and Cordyceps have been recommended for patients with DKD in the practice guidelines of Chinese medicine. ${ }^{13}{ }^{14}$ However, these guidelines were based on experts' consensus rather than outcomes of systematically evaluated best available clinical evidence. Moreover, safety concerns exist due to the potential for aristolochic-acid nephrotoxicity with some herbal products. ${ }^{5}{ }^{15}$ Even though legislation and quality control have been reinforced in recent years, the general lack of information regarding the safety profiles of herbal formulae due to their multi-compound nature have limited their application. ${ }^{516}$

In recent years, there have been a growing number of clinical trials and systematic reviews of CHM for DKD but not of placebo-controlled trials. We therefore undertook a systematic review and meta-analysis of randomised, placebo-controlled trials to evaluate the efficacy and safety of oral CHM as adjunctive treatment for DKD.

\section{METHODS}

This systematic review was conducted following the Cochrane Handbook of Systematic Reviews of Interventions and reported in accord with the Preferred Reporting Items for Systematic Reviews and Meta-Analyses guidelines. ${ }^{1718}$ The protocol was registered in the PROSPERO database and can be accessed online.

\section{Search strategy}

A comprehensive search was conducted in the following databases irrespective of publication status or language: MEDLINE, EMBASE, Cumulative Index of Nursing and Allied Health Literature, Cochrane Central Register of
Controlled Trials (CENTRAL), Allied and Complementary Medicine Database, China BioMedical Literature, China National Knowledge Infrastructure, Chonqing VIP and Wanfang. The former five databases were in English while the later four were in Chinese. Databases were searched from inception to May 2018. The US National Institutes of Health register (ClinicalTrials.gov), the Australian New Zealand Clinical Trial Registry, the Chinese Clinical Trial Registry and the European Union Clinical Trials Register were searched for completed but unpublished trials. Furthermore, reference lists of related systematic reviews were reviewed for additional publications.

Search terms included 'diabetic nephropathy', 'diabetic kidney disease', 'albuminuria', 'Traditional Chinese Medicine', 'randomised controlled trial' and their synonyms. All terms were mapped to controlled vocabulary (where applicable) in addition to being searched as keywords. The MEDLINE search strategy is provided in online table S1.

\section{Eligibility criteria}

Eligible studies had to fulfil the following criteria: (1) RCT design; (2) included primary diabetes adults with persistent albuminuria/proteinuria, which was defined as an albumin excretion rate (AER) more than $20 \mu \mathrm{g}$ / min, an albumin-to-creatinine ratio (ACR) larger than $30 \mathrm{mg} / \mathrm{g}^{45}$ or 24 hours proteinuria over $0.5 \mathrm{~g} /$ day (the overt DKD stage defined by Mogensen and used as in DKD diagnostic criteria in China ${ }^{1920}$; (3) oral CHM as intervention, which could have been either single or multiple ingredients in any form (decoction, granules, capsules and so on); (4) CHM matched placebo was applied in the control group; (5) both intervention and control groups received the same conventional treatments of DKD, including comprehensive management of glycaemia, blood pressure, serum lipid level, life-style and nutrition in accordance with Kidney Disease Outcomes Quality Initiative clinical practice guidelines' recommendation $^{45}$ and (6) the study reported at least one of the primary outcomes. Studies including patients with albuminuria that was not caused by diabetes, patients who already had ESKD, or those receiving renal replacement therapy were excluded.

\section{Outcomes of interest}

Primary outcomes of interest included albuminuria/ proteinuria, kidney function, number of participants progressing to ESKD, all-cause mortality and adverse events, at the end of treatment or follow-up. Progression to ESKD was defined as initiation of renal replacement therapy or estimated glomerulus filtration rate (eGFR) lower than $15 \mathrm{~mL} / \mathrm{min} / 1.73 \mathrm{~m}^{2}$. Kidney function was reflected by the measurement of serum creatinine (Scr) concentration and GFR. Likewise, quantitative measurement of albuminuria and proteinuria included urinary AER, ACR, 24 hours urine protein (UP) excretion and protein-to-creatinine ratio. 
Secondary outcomes included cardiovascular mortality, all-cause hospitalisation, quality of life (QoL) measured by validated scales, indicators of risk factor control (such as fasting blood glucose, glycated haemoglobin [HbAlc], blood pressure, total cholesterol, triglycerides, low-density lipoprotein cholesterol [LDL-C] and high-density lipoprotein cholesterol [HDL-C]). All outcomes were reported with specified units at the end of treatment or at the end of follow-up.

Safety outcomes included numbers and type of adverse events and serious adverse events during the study period.

\section{Study selection and data extraction}

Titles and abstracts identified in searching were screened by one reviewer and then checked by another (LaZ and $\mathrm{XQ})$ against the predefined criteria. After titles and abstracts screening, possibly relevant studies underwent full-text review by LaZ and cross checked by LY to confirm their eligibility. Any disagreement was resolved by consensus and discussion with a third reviewer (JS or ALZ).

Two reviewers ( $\mathrm{LaZ}$ and LY) independently extracted data from eligible studies into a pre-designed spreadsheet. A third reviewer (JS) cross-checked the data. Study design characteristics, trial locations, demographic features (age, types of diabetes, baseline albuminuria, kidney function and so on), intervention and control protocol (herbal ingredients, dosage, frequency, treatment duration, follow-up period and so on), and outcome measures were recorded. Authors of studies with missing data were contacted by email or telephone to obtain additional data.

\section{Data synthesis and analysis}

All studies satisfying the eligibility criteria were included for qualitative synthesis. For continuous variables, mean and SD of each study were obtained and pooled as mean difference (MD) or standardised mean differences (SMD) with a $95 \%$ confidence interval (CI). SMD was used in the meta-analysis of albuminuria and proteinuria outcomes due to the different scales used in the included studies such as microgram per minute $(\mu \mathrm{g} / \mathrm{min})$, milligram to gram $(\mathrm{mg} / \mathrm{g}$ ) and milligram per day (mg/24hours). For dichotomous data, risk ratios (RR) were calculated with a $95 \%$ CI. Considering the diversity of interventions and potential heterogeneity among included studies, a random-effect model was applied in all meta-analyses. Review Manager Software (RevMan V.5.3) was used to perform the statistical analysis. ${ }^{21}$

Predefined subgroup analysis included baseline DKD severity and CHM formulae. Heterogeneity between studies was detected by using the Cochrane $Q$ statistic and $\mathrm{I}^{2}$ test. For outcomes with substantial heterogeneity $\left(\mathrm{I}^{2}\right.$ levels $>50 \%$ ), subgroup analyses were performed to explore potential sources, whereby results were stratified by factors, such as different measured approaches for the same outcome. Sensitivity analysis was performed by excluding studies with high/unclear risk-of-bias (ROB) in the domain of random sequence generation. Publication bias was explored when 10 or more studies were included in one meta-analysis by visual inspection of funnel plots for asymmetry.

\section{Quality assessment}

The methodologic quality of each individual study was assessed by two reviewers ( $\mathrm{LaZ}$ and LY) in parallel according to the Cochrane ROB tool. ${ }^{22}$ For the domain of other sources of bias, baseline imbalance and conflicts of interest were evaluated. Each domain was judged as high, low or unclear ROB with justifications. The consistency was checked by a third reviewer (LeiZ) and disagreements were resolved by discussion with methodologists (ALZ and XG).

To evaluate the overall quality of evidence for primary outcomes, the Grading of Recommendations Assessment, Development and Evaluation (GRADE) approach was applied. ${ }^{23}$ A panel group was formed to make the GRADE evaluation, which included methodologists, CM practitioners and conventional medicine physicians. The assessments of evidence started at 'high quality', and were downgraded when significant ROB, indirectness, inconsistency, imprecision of estimated effect or publication bias were detected.

\section{Patient and public involvement}

Patients or public were not directly involved in this systematic review.

\section{RESULTS \\ Description of studies}

The comprehensive search retrieved over 50 thousand citations and 7255 of them were examined in fulltext (figure 1). Eighty-five percent of the studies were excluded due to lack of a placebo control. As a result, 20 eligible studies with 23 publications involving 2719 DKD participants were included. ${ }^{24-46}$ For studies with multiple reports, the most recent publication or the one with primary outcomes was used, and complementary outcomes data from other reports were extracted and merged.

Characteristics of the included studies are summarised in table 1. All 20 studies were conducted in China. Except for one study ${ }^{24}$ written in English, all others were published in Chinese language between 2000 and 2017. Enrolled participants were all diabetic patients with persistent albuminuria or proteinuria but varied in terms of baseline kidney function. The mean of age was 55.1 years old (range 20-79 years). Three studies ${ }^{34} 3537$ used herbal compounds or a single herb as intervention while the remaining 17 studies used CHM formulae with multi-ingredients. The ingredients of CHM used in each study are provided in table 2 . The most common herbal ingredients used by ten or more studies was Astragali radix, Rehmanniae Radix and Rhei Radix et Rhizoma. All studies applied CHM matched placebo, except for 


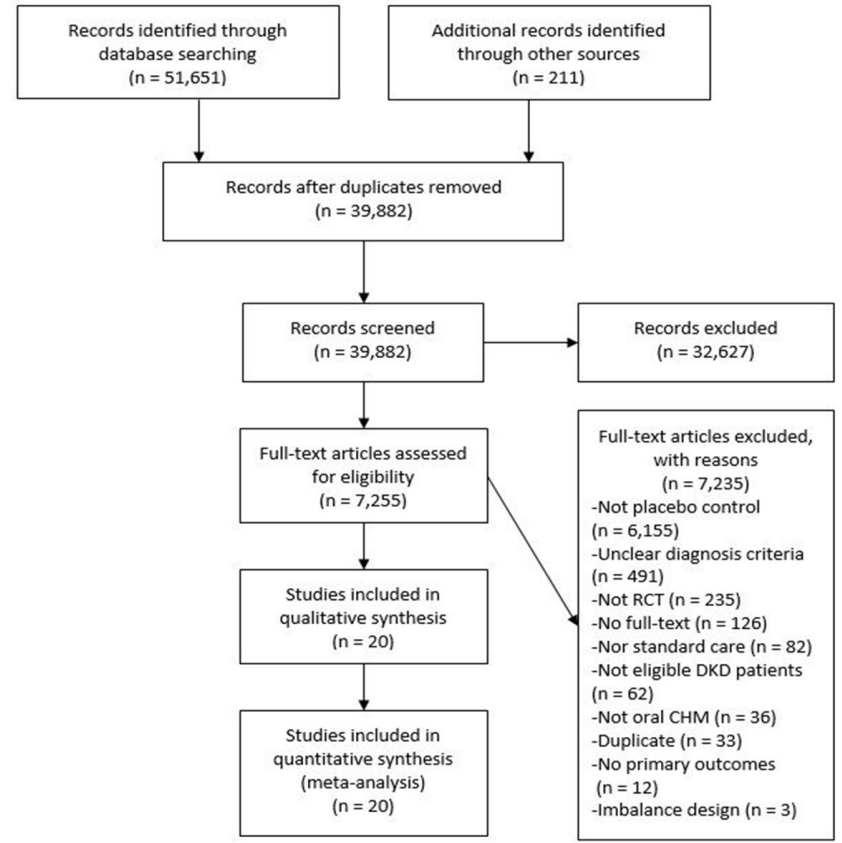

Figure 1 Preferred Reporting Items for Systematic Reviews and Meta-Analyses flowchart of searching and screening.

one $^{32}$ which made Captopril (comparator) identical in appearance to CHM (intervention). Treatment duration ranged from 4 weeks to 2 years (median 3 months). There were no outcome data with respect to cardiovascular mortality and all-cause hospitalisation among the included studies.

\section{Quality of studies}

Generally, the quality of included studies was fair with low or unclear ROB, especially regarding blinding and outcome data completeness (figure 2). Two studies were judged at high ROB with respect to blinding of patients and personnel because blinding may have been compromised by prescription of unequal numbers/amounts of medication between groups. ${ }^{28} 31$ Twelve studies reported correct procedures for random sequence generation, ${ }^{24} 25$ 28-30 34-36 384144 whereas eight studies did not provide adequate details. For the domain of allocation concealment, one study did not conceal the allocation to researchers thus was judged at high ROB. ${ }^{41}$ Seven studies were considered at high risk of selection reporting bias (mainly incomplete reporting in secondary outcomes), 25262931333844 while 13 studies were at unclear risk because protocols were not found. Other bias assessment included baseline balance and conflict of interest. Two studies included pharmaceutical industry employees as coauthors without statements regarding their roles in the study, thereby these two trials were judged as high risk for potential conflicts of interest. ${ }^{34}{ }^{35}$ Seven studies without baseline statistical test results or without information regarding sources of funding were judged to be at unclear risk. 26283136373940

\section{Effecacy of Chinese herbal medicine}

Considering the uses of RAS blockage may affect the primary outcomes, studies were categorised and separated into three groups according to trial application of RAS blockade (ACE inhibitors [ACEi] and/or angiotensin receptor blockers [ARB]) in each arm prior to meta-analysis. It should be noted that conventional concurrent treatments of DKD recommended by guidelines were applied equally in both groups in all included studies, such that these conventional treatments are not separately mentioned henceforth. The three groups were: - CHM versus placebo $2530343537-4045$.

- CHM plus ACEi/ARB versus placebo plus ACEi/ $\mathrm{ARB}^{24} 33364144$.

- CHM versus placebo plus ACEi/ARB. ${ }^{26-29} 31324446$

Mortality and progression to ESKD

Though all-cause mortality was measured in a study ${ }^{31}$ comparing CHM with matched placebo plus Irbesartan, no deaths were observed among the 315 participants during the 2-year follow-up (table 3). Within the same trial, the number of patients that progressed to ESKD was reported as part of a composite outcome, measuring the number of patients with microalbuminuria progressing to macroalbuminuria, doubling Scr from baseline or initiating dialysis. Compared with placebo plus Irbesartan, the risk of experiencing this composite outcome may be $66 \%$ lower in the CHM group over 2-year period (RR: $0.34,95 \%$ CI [ 0.15 to 0.77$], \mathrm{p}=0.01$; low-quality evidence).

\section{Albuminuria}

Fourteen studies reported albuminuria at the end of treatment (figure 3A). Based on meta-analysis of eight studies $^{25} 30343537394045$ involving 1021 participants, use of CHM probably lowered albuminuria compared with placebo over 2-12 months intervention (SMD -0.92, $95 \%$ CI $[-1.35$ to -0.51$], \mathrm{I}^{2}=87 \%, \mathrm{p}<0.0001$; moderate quality evidence). Subgroup analysis suggested different CHM formulae could be the sources of heterogeneity (online table S2). The estimate of effect with the least heterogeneity was observed in the Qi Wei granule CHM subgroup $^{3045}$ in which albuminuria was $70.06 \mathrm{mg} / 24$ hours lower compared with placebo after 3 months (95\% CI [-88.84 to -51.28$\left.], \mathrm{I}^{2}=0 \%, \mathrm{p}<0.0001\right)$. Likewise, the Arctiin granule ${ }^{34} 35$ reduced albuminuria more than placebo after 2 months intervention (SMD $-0.38,95 \%$ CI $[-0.56$ to -0.20$\left.], \mathrm{I}^{2}=0 \%, \mathrm{p}<0.0001\right)$.

When used in combination with ACEi/ARB, slightly lower end of treatment albuminuria level was still observed in the CHM rather than in the placebo group over a 3-6 month intervel (SMD $-0.56,95 \%$ CI [-1.04 to -0.08$], \mathrm{I}^{2}=64 \%, \mathrm{p}=0.002$; moderate quality evidence). ${ }^{24} 3641$ Although lower albuminuria excretion was observed in the CHM group, ${ }^{262732}$ the effect of CHM in decreasing albuminuria compared with ACEi/ARB was uncertain because of the very low quality of evidence (table 3). 
Table 1 Characteristic of included studies

\begin{tabular}{|c|c|c|c|c|c|c|c|}
\hline $\begin{array}{l}\text { Study } \\
\text { (first author, year) }\end{array}$ & $\begin{array}{l}\text { Sample size } \\
\text { (M/F) }\end{array}$ & Age & $\begin{array}{l}\text { Diabetes } \\
\text { type }\end{array}$ & $\begin{array}{l}\text { Inclusion criteria of kidney } \\
\text { function }\end{array}$ & $\begin{array}{l}\text { Intervention and control } \\
\text { protocol }\end{array}$ & $\begin{array}{l}\text { Duration } \\
\text { (months) }\end{array}$ & Reported outcomes \\
\hline Fan, $2010^{25}$ & $\begin{array}{l}61 \\
(28 / 33)\end{array}$ & 59.6 & 2 & $\begin{array}{l}\text { Albuminuria } 30-300 \mathrm{mg} / \mathrm{g} \text { or } \\
30-300 \mathrm{mg} / 24 \text { hours }\end{array}$ & $\begin{array}{l}\text { T: Qi Kui granule } 1 \text { bag } \\
\text { two times daily } \\
\text { C: placebo }\end{array}$ & 12 & UAE; FBG \\
\hline Jia, $2012^{30}$ & $\begin{array}{l}60 \\
(29 / 31)\end{array}$ & 58.3 & 2 & $\begin{array}{l}\text { Proteinuria }<3.5 \mathrm{~g} / 24 \text { hours; } \\
\text { Normal Scr level }\end{array}$ & $\begin{array}{l}\text { T: Qi Wei granule } 4.5 \mathrm{~g} \text { two } \\
\text { times daily } \\
\text { C: placebo }\end{array}$ & 3 & UAE; 24h UP; \\
\hline
\end{tabular}

\begin{tabular}{|c|c|c|c|c|c|c|c|}
\hline $\mathrm{Ma}, 2011 \mathrm{a}^{34}$ & $\begin{array}{l}414 \\
(186 / 228)\end{array}$ & 56.6 & NS & $\begin{array}{l}\text { Proteinuria } \leq 4.5 \mathrm{~g} / 24 \text { hours; } \\
\text { Scr } \leq 190 \mu \mathrm{mol} / \mathrm{L}\end{array}$ & $\begin{array}{l}\text { T: Arctiin granule } 1 \text { bag two } \\
\text { times daily } \\
\text { C: placebo }\end{array}$ & 2 & UAE; 24h UP; \\
\hline
\end{tabular}

\begin{tabular}{|c|c|c|c|c|}
\hline Ma, 2011b & $\begin{array}{l}186 \\
(78 / 108)\end{array}$ & 55.3 & NS & $\begin{array}{l}\text { Proteinuria } \leq 3.5 \mathrm{~g} / 24 \text { hours; } \\
\text { Scr }<176 \mu \mathrm{mol} / \mathrm{L}\end{array}$ \\
\hline
\end{tabular}

T1: Arctiin granule 2 bag $2 \quad$ UAE; 24h UP;

two times daily+placebo 2

bag qd

T2: Arctiin granule 1 bag three

times a day + placebo 1 bag

three times a day

C: placebo 2 bag three times a day

\begin{tabular}{|c|c|c|c|c|c|c|c|}
\hline Wei, $2012^{37}$ & $\begin{array}{l}56 \\
(24 / 32)\end{array}$ & 50.6 & NS & $\begin{array}{l}\text { Albuminuria } 30- \\
300 \mathrm{mg} / 24 \text { hours; } \\
\text { Scr } \leq 105 \mu \mathrm{mol} / \mathrm{L}\end{array}$ & $\begin{array}{l}\text { T: Xue Zhi Kang capsule } 0.6 \mathrm{~g} \\
\text { three times a day } \\
\text { C: placebo }\end{array}$ & 3 & $\begin{array}{l}\text { UAE; TC; TG; LDLC; } \\
\text { HDLC }\end{array}$ \\
\hline Wei, $2016^{38}$ & $\begin{array}{l}41 \\
(32 / 9)\end{array}$ & 61.8 & 2 & $\begin{array}{l}\text { Albuminuria }>30 \mathrm{mg} / \mathrm{g} \text { and } \\
\text { Proteinuria } \leq 3.5 \mathrm{~g} / 24 \text { hours } \\
\text { GFR } \geq 30 \mathrm{~mL} / \mathrm{min}\end{array}$ & $\begin{array}{l}\text { T: Gan Di capsule 3\# three } \\
\text { times a day } \\
\text { C: placebo }\end{array}$ & 6 & $\begin{array}{l}\text { Scr; FBG; A1C; TC; TG; } \\
\text { LDLC; HDLC }\end{array}$ \\
\hline Xie, $2011^{39}$ & $\begin{array}{l}67 \\
(30 / 37)\end{array}$ & 62.3 & 2 & Albuminuria $30-299 \mu \mathrm{g} / \mathrm{mg}$ & $\begin{array}{l}\text { T: Liu Wei Di Huang pill } \\
3 \mathrm{~g} \text { three times a day } \\
\text { +Ginkgo biloba tablet } 19.2 \mathrm{mg} \\
\text { three times a day } \\
\text { C: LWDHW Placebo+GBT } \\
\text { placebo }\end{array}$ & 24 & $\begin{array}{l}\text { UAE; FBG; A1C; TC; TG; } \\
\text { LDLC; HDLC; SBP; DBP }\end{array}$ \\
\hline Yang, $2014^{40}$ & $\begin{array}{l}142 \\
(80 / 62)\end{array}$ & 48.5 & NS & $\begin{array}{l}\text { Albuminuria } 30- \\
300 \text { mg/24 hours; } \\
\text { Normal Scr level }\end{array}$ & $\begin{array}{l}\text { T: Qi Ming granule } 4.5 \mathrm{~g} \text { three } \\
\text { times a day } \\
\text { C: placebo }\end{array}$ & 3 & $\begin{array}{l}\text { UAE; FBG; TC; TG; } \\
\text { LDLC; HDLC }\end{array}$ \\
\hline Zhou, $2014^{45}$ & $\begin{array}{l}48 \\
(27 / 21)\end{array}$ & 58.5 & 2 & $\begin{array}{l}\text { Proteinuria } \leq 3.5 \mathrm{~g} / 24 \text { hours; } \\
\text { Normal Scr level }\end{array}$ & $\begin{array}{l}\text { T: Qi Wei granule } 6 \mathrm{~g} \text { three } \\
\text { times a day } \\
\text { C: placebo }\end{array}$ & 3 & $\begin{array}{l}\text { UAE; } 24 h \text { UP; Scr; GFR; } \\
\text { FBG; A1C; SBP; DBP }\end{array}$ \\
\hline $\mathrm{Li}, 2015^{24}$ & $\begin{array}{l}180 \\
(100 / 80)\end{array}$ & 59.0 & 2 & $\begin{array}{l}\text { Albuminuria }>20 \mu \mathrm{g} / \mathrm{min} \text { or } \\
\text { Proteinuria } 0.5-2 \mathrm{~g} / 24 \text { hours } \\
\text { GFR } 60-130 \mathrm{~mL} / \mathrm{min}\end{array}$ & $\begin{array}{l}\text { T: Tang Shen granule } 8 \mathrm{~g} \\
\text { two times daily+ACEi/ARB } \\
\text { C: placebo+ACEi/ARB }\end{array}$ & 6 & $\begin{array}{l}\text { UAE; 24h UP; Scr; GFR; } \\
\text { A1C; TC; TG; LDLC; } \\
\text { HDLC; SBP; DBP; QoL }\end{array}$ \\
\hline Liu, $2015^{33}$ & $\begin{array}{l}60 \\
(\mathrm{NS})\end{array}$ & $20-70$ & 2 & $\begin{array}{l}\text { Albuminuria } 20-200 \mu \mathrm{g} / \mathrm{min} \text { or } \\
\text { Proteinuria } \leq 3.5 \mathrm{~g} / 24 \text { hours } \\
\text { GFR }>60 \mathrm{~mL} / \mathrm{min}\end{array}$ & $\begin{array}{l}\text { T: Qi Huang capsule } 1.9 \mathrm{~g} \\
\text { three times daily+losartan } \\
\text { C: placebo+losartan }\end{array}$ & 6 & 24h UP; Scr \\
\hline $\mathrm{Ni}, 2013^{36}$ & $\begin{array}{l}224 \\
(112 / 112)\end{array}$ & 54.7 & NS & $\begin{array}{l}\text { Albuminuria } 20-200 \mu \mathrm{g} / \mathrm{min} \text { or } \\
\text { Proteinuria } \leq 3.5 \mathrm{~g} / 24 \text { hours } \\
\text { GFR } 60-130 \mathrm{~mL} / \mathrm{min}\end{array}$ & $\begin{array}{l}\text { T: Qi Yao Xiao Ke } \\
\text { capsule } 2.4 \mathrm{~g} \text { three times } \\
\text { daily+benazepril } \\
\text { C: placebo+benazepril }\end{array}$ & 3 & $\begin{array}{l}\text { UAE; 24h UP; Scr; GFR; } \\
\text { FBG; A1C; TC; TG; } \\
\text { LDLC; HDLC }\end{array}$ \\
\hline Yang, $2017^{41}$ & $\begin{array}{l}25 \\
(23 / 2)\end{array}$ & 59.3 & 2 & $\begin{array}{l}\text { Albuminuria } 20-200 \mu \mathrm{g} / \mathrm{min} \text { or } \\
30-300 \mathrm{mg} / 24 \text { hours }\end{array}$ & $\begin{array}{l}\text { T: Qi Zhu granule } 1 \text { bag } \\
\text { two times daily+irbesartan } \\
\text { C: placebo+irbesartan }\end{array}$ & 6 & $\begin{array}{l}\text { UAE; Scr; GFR FBG; } \\
\text { A1C; TC; TG; LDLC; } \\
\text { HDLC }\end{array}$ \\
\hline Zhang, $2006^{42-44}$ & $\begin{array}{l}221 \\
(119 / 102)\end{array}$ & 61.9 & NS & $\begin{array}{l}\text { Proteinuria }<10 \mathrm{~g} / 24 \text { hours; } \\
\text { Scr } 133-354 \mu \mathrm{mol} / \mathrm{L} \text { or } \mathrm{Ccr} \\
30-70 \mathrm{~mL} / \mathrm{min}\end{array}$ & $\begin{array}{l}\text { T 1: Modified Qi Wei granule } 1 \\
\text { bag two times daily+losartan } \\
\text { T 2: Modified Qi Wei granule } 1 \\
\text { bag two times daily+losartan } \\
\text { simulant } \\
\text { C: placebo+losartan }\end{array}$ & 3 & 24h UP; Scr; GFR; QoL \\
\hline Gao, $2006^{26}$ & $\begin{array}{l}90 \\
(\mathrm{NS})\end{array}$ & $35-70$ & 2 & $\begin{array}{l}\text { Albuminuria } 20-200 \mu \mathrm{g} / \mathrm{min} \text { or } \\
30-300 \mathrm{mg} / 24 \text { hours }\end{array}$ & $\begin{array}{l}\text { T: Tang Shen Ning granule } 5 \mathrm{~g} \\
\text { three times daily+benazepril } \\
\text { simulant } \\
\text { C: placebo+benazepril }\end{array}$ & 2 & UAE; Scr; \\
\hline Gao, $2017^{27}$ & $\begin{array}{l}250 \\
(116 / 134)\end{array}$ & 52.3 & 2 & $\begin{array}{l}\text { Albuminuria } 30- \\
300 \mathrm{mg} / 24 \text { hours }\end{array}$ & $\begin{array}{l}\mathrm{T} \text { : Tang Shen Ning granule } \\
8 \mathrm{~g} \text { three times daily+losartan } \\
\text { simulant } \\
\text { C: placebo+losartan }\end{array}$ & 3 & UAE; Scr; FBG; A1C \\
\hline
\end{tabular}

Continued 


\begin{tabular}{|c|c|c|c|c|c|c|c|}
\hline $\begin{array}{l}\text { Study } \\
\text { (first author, year) }\end{array}$ & $\begin{array}{l}\text { Sample size } \\
\text { (M/F) }\end{array}$ & Age & $\begin{array}{l}\text { Diabetes } \\
\text { type }\end{array}$ & $\begin{array}{l}\text { Inclusion criteria of kidney } \\
\text { function }\end{array}$ & $\begin{array}{l}\text { Intervention and control } \\
\text { protocol }\end{array}$ & $\begin{array}{l}\text { Duration } \\
\text { (months) }\end{array}$ & Reported outcomes \\
\hline Han, $2014^{28}$ & $\begin{array}{l}104 \\
\text { (NS) }\end{array}$ & $30-78$ & 2 & $\begin{array}{l}\text { Proteinuria } \geq 0.5 \mathrm{~g} / 24 \text { hours } \\
\text { Scr }<265 \mu \mathrm{mol} / \mathrm{L}\end{array}$ & $\begin{array}{l}\text { T1: Bao Shen pill } \\
1 \text { bag two times } \\
\text { daily+Tripterygium glycosides } \\
20 \text { mg two times daily } \\
\text { T2: Bao Shen pill } 1 \text { bag } \\
\text { two times daily } \\
\text { C: BS placebo+valsartan }\end{array}$ & 1 & 24h UP; Scr \\
\hline Jia, $2015^{29}$ & $\begin{array}{l}56 \\
(31 / 25)\end{array}$ & 59.6 & NS & $\begin{array}{l}\text { Proteinuria }<10 \mathrm{~g} / 24 \text { hours; } \\
\text { Scr }<265 \mu \mathrm{mol} / \mathrm{L}\end{array}$ & $\begin{array}{l}\text { T: San Huang Yi Shen } \\
\text { granule } 1 \text { bag two times } \\
\text { daily+irbesartan simulant } \\
\text { C: placebo+irbesartan }\end{array}$ & 3 & GFR \\
\hline Li, $2012^{3146}$ & $\begin{array}{l}315 \\
(194 / 121)\end{array}$ & 58.1 & NS & $\begin{array}{l}\text { Proteinuria }<10 \mathrm{~g} / 24 \text { hours; } \\
\mathrm{Scr}<265 \mu \mathrm{mol} / \mathrm{L} \text { or } \\
\text { GFR }>40 \mathrm{~mL} / \mathrm{min} \text {; }\end{array}$ & $\begin{array}{l}\text { T: Modified Qi Wei granule } \\
4.5 \mathrm{~g} \text { two times daily } \\
\text { C: placebo+irbesartan }\end{array}$ & 24 & $\begin{array}{l}\text { Mortality; Composite } \\
\text { endpoints; QoL }\end{array}$ \\
\hline Lin, $2000^{32}$ & $\begin{array}{l}119 \\
(46 / 73)\end{array}$ & 55.3 & NS & $\begin{array}{l}\text { Proteinuria }<0.5 \mathrm{~g} / 24 \text { hours; } \\
\text { Normal Scr level }\end{array}$ & $\begin{array}{l}\text { T: Tang Wei Kang capsule } 2 \mathrm{~g} \\
\text { three times daily } \\
\text { C: Captopril (same } \\
\text { appearance as herbal } \\
\text { capsule) }\end{array}$ & 3 & $\begin{array}{l}\text { UAE; FBG; A1C; TC; TG; } \\
\text { HDLC }\end{array}$ \\
\hline
\end{tabular}

24h UP, 24 hours proteinuria; A1C, glycated haemoglobin; C, control group; Ccr, creatinine clearance rate; DBP, diastolic blood pressure; FBG, fasting blood glucose; GFR, glomerular filtration rate; HLDL-C, high-density lipoprotein cholesterol; LDLC, low-density lipoprotein cholesterol; M/F, male versus female; NS, not specified in the original reports; qd, once daily; QoL, quality of life; SBP, systolic blood pressure; Scr, serum creatinine concentration; T, tested group; TC, total cholesterol; TG, triglycerides; UAE, urinary albuminuria excretion.

\section{Proteinuria}

Nine studies measured end of treatment 24 hours proteinuria (figure $3 \mathrm{~B}$ ). The pooled estimated effect showed CHM may reduce proteinuria compared with placebo after 2-3 months intevention, although heterogeneity was high (SMD $-1.34,95 \%$ CI $[-2.18$ to -0.51$], \mathrm{I}^{2}=94 \%$, $\mathrm{p}=0.002$; low quality evidence).$^{30343545}$ Subgroup analysis revealed that different formulae and proteinuria scales may have been the source of heterogeneity (online table S2). Pooled estimates of effect of Qi Wei granule ${ }^{3045}$ and Arctiin granule $\mathrm{e}^{3435}$ both showed that CHM may lead to greater reductions in proteinuria than placebo. Subgroup of measurements unit of milligram per 24 hours showed the proteinuria was $324.42 \mathrm{mg} / 24$ hours lower $(95 \% \mathrm{CI}$, $[-485.15$ to -163.69$\left.] ; \mathrm{I}^{2}=30 \% ; \mathrm{p}<0.0001\right)$ in the CHM group than the placebo group. 343545

When used in combination with ACEi/ARB, meta-analysis of four studies with 489 participants ${ }^{24} 46$ showed that CHM may make little or no difference to proteinuria compared with placebo after 3-6 months of intervetions (SMD $-0.15,95 \%$ CI $[-0.52$ to 0.23$], \mathrm{I}^{2}=72 \%, \mathrm{p}=0.44$; low quality evidence). Sources of heterogeneity were not identified (online table S2). Likewise, low quality evidence suggested that CHM may make no differences to end of treatment proteinuria compared with placebo plus ACEi/ARB after 1-3 months intervention (table 3). ${ }^{28} 44$

\section{Serum creatinine level}

Ten studies provided end of treatment data of Scr level (figure 3C). Pooled estimation of two small studies 3845 showed that the additional CHM intervention probably made little difference to Scr levels compared with placebo after 3-6 months (MD $5.75 \mu \mathrm{mol} / \mathrm{L}, 95 \%$ CI [-2.06 to 13.57], $\mathrm{I}^{2}=0 \%, \mathrm{p}=0.15$; moderate quality evidence). When used in combination with ACEi/ARB, end of treatment Scr level was slightly lower in the CHM group compared with the placebo group over 3-6 months, but was not clinically significant (MD $-4.02 \mu \mathrm{mol} / \mathrm{L}, 95 \%$ CI [-7.81 to -0.23$]$, $\mathrm{I}^{2}=0 \%, \mathrm{p}=0.15$; moderate quality evidence). ${ }^{24} 33364144$ Subgroup analysis found that the lowering Scr effect of CHM was evident in patients with abnormal baseline $\mathrm{Scr}$ after 3 months intevention (MD -9.99 $\mathrm{mol} / \mathrm{L}, 95 \%$ CI $[-17.71$ to -2.26$\left.], \mathrm{I}^{2}=0 \%, \mathrm{p}=0.01\right) .^{3644}$

Slightly lower Scr levels were observed in the CHM group compared with placebo plus ACEi/ARB group after 1-3 months intervention, but the difference was not clinically significant. ${ }^{26-2844}$ A similar effect was found in the subgroup analysis of Tang Shen Ning formula compared with placebo plus ARB after 2-3 months treatment $\left(\mathrm{MD}-3.96 \mu \mathrm{mol} / \mathrm{L}, 95 \%\right.$ CI $[-6.13$ to -1.78$], \mathrm{I}^{2}=6 \%$, $\mathrm{p}=0.0004) .^{2627}$

\section{Estimated glomerular filtration rate}

Of the eight studies, the glomerular filtration rate (GFR) was estimated by either Cockcroft-Gault equation or other Scr-based equations (figure 3D). When used in combination with ACEi/ARB, the end of treatment eGFR was slightly higher in the CHM group compared with placebo group after 3-6 intervention (MD $6.28 \mathrm{~mL} / \mathrm{min}$, $95 \%$ CI [2.42 to 10.14$], \mathrm{I}^{2}=0 \%, \mathrm{p}=0.001$; moderate quality evidence). ${ }^{24364144}$ Subgroup analysis of specific formula showed that the end of treatment eGFR was $5.22 \mathrm{~mL} /$ min higher ( $95 \%$ CI $[0.69$ to 9.74$\left.], \mathrm{I}^{2}=0 \%, \mathrm{p}=0.02\right)$ in the Tang Shen Fang formula plus ACEi/ARB group than the placebo plus ACEi/ARB group. ${ }^{24} 44$ It should be noted that Cockcroft-Gault equation may overestimate eGFR, leading to $10 \%-20 \%$ higher value in pooled estimation 
Table 2 Herbal ingredients used in included studies

\begin{tabular}{|c|c|c|}
\hline $\begin{array}{l}\text { Study } \\
\text { (first author, year) }\end{array}$ & Formulae name & Ingredients \\
\hline Fan, $2010^{25}$ & Qi Kui granule & Astragali Radix; Polygoni Multiflori Radix; Abelmoschi Corolla \\
\hline $\mathrm{Jia}, 2012^{30}$ & Qi Wei granule & $\begin{array}{l}\text { Astragali Radix; Rehmanniae Radix; Rhei Radix et Rhizoma; Prunellae Spica; Curcumae } \\
\text { Rhizoma; Euonymus Alatus; Notoginseng Radix et Rhizoma }\end{array}$ \\
\hline Ma, $2011 a^{34}$ & Arctiin granule & Arctii Fructus \\
\hline Ma, $2011 b^{35}$ & Arctiin granule & Arctii Fructus \\
\hline Wei, $2012^{37}$ & Xue Zhi Kang capsule & Fermentum Rubrum* \\
\hline Wei, $2016^{38}$ & Gan Di capsue & $\begin{array}{l}\text { Scutellariae Radix; Astragali Radix; Corni Fructus; Rehmanniae Radix Phylianthi Fructus; } \\
\text { Leonuri Herba Leonuri Herba; Bombyx Batryticatus; Sophorae Flos (stir fry processed) }\end{array}$ \\
\hline Xie, $2011^{39}$ & $\begin{array}{l}\text { Liu Wei Di Huang pill } \\
\text { Ginkgo biloba tablet }\end{array}$ & $\begin{array}{l}\text { Rehmanniae Radix; Corni Fructus; Dioscoreae Rhizoma; Alismatis Rhizoma; Moutan Cortex; } \\
\text { Poria; Ginkgo Folium }\end{array}$ \\
\hline Yang, $2014^{40}$ & Qi Ming granule & $\begin{array}{l}\text { Astragali Radix; Puerariae Lobatae Radix; Rehmanniae Radix; Lycii Fructus; Cassiae Semen; } \\
\text { Leonuri Fructus; Typhae Pollen; Hirudo }\end{array}$ \\
\hline Zhou, $2014^{45}$ & Qi Wei granule & $\begin{array}{l}\text { Astragali Radix; Rehmanniae Radix; Rhei Radix et Rhizoma; Prunellae Spica; Curcumae } \\
\text { Rhizoma; Euonymus Alatus; Notoginseng Radix et Rhizoma }\end{array}$ \\
\hline $\mathrm{Li}, 2015^{24}$ & Tang Shen granule & $\begin{array}{l}\text { Astragali Radix; Rehmanniae Radix; Rhei Radix et Rhizoma; Notoginseng Radix et Rhizoma; } \\
\text { Euonymus Alatus; Corni Fructus; Aurantii Fructus }\end{array}$ \\
\hline Liu, $2015^{33}$ & Qi Huang capsule & $\begin{array}{l}\text { Astragali Radix; Rehmanniae Radix; Ligustri Lucidi Fructus; Hirudo; Bombyx Batryticatus; } \\
\text { Eupolyphaga Steleophaga; Rhei Radix et Rhizoma; Gymnema sylvestre*; Sinomenii Caulis; } \\
\text { Plantaginis Semen }\end{array}$ \\
\hline $\mathrm{Ni}, 2013^{36}$ & Qi Yao Xiao Ke capsule & $\begin{array}{l}\text { Panacis Quinquefolii Radix; Astragali Radix; Rehmanniae Radix; Dioscoreae Rhizoma; Corni } \\
\text { Fructus; Lycii Fructus; Ophiopogonis Radix; Anemarrhenae Rhizoma; Trichosanthis Radix; } \\
\text { Puerariae Lobatae Radix; Schisandrae Chinensis Fructus Schisandrae Chinensis Fructus; Galla } \\
\text { Chinensis }\end{array}$ \\
\hline Yang, $2017^{41}$ & Qi Zhu granule & $\begin{array}{l}\text { Astragali Radix; Ligustri Lucidi Fructus; Atractylodis Macrocephalae Rhizoma; Abelmoschi } \\
\text { Corolla; Rosae laevigatae Fructus Dioscoreae Spongiosae Rhizoma; Paeoniae Radix Rubra; } \\
\text { Coptidis Rhizoma }\end{array}$ \\
\hline Zhang, 2006 & Modified Qi Wei granule & $\begin{array}{l}\text { Astragali Radix; Rehmanniae Radix; Prunellae Spica; Rhei Radix et Rhizoma; Euonymus Alatus; } \\
\text { Epimedii Folium; Corni Fructus; Curcumae Longae Rhizoma }\end{array}$ \\
\hline Gao, $2006^{26}$ & Tang Shen Ning granule & $\begin{array}{l}\text { Astragali Radix; Rehmanniae Radix; Euryales Semen; Corni Fructus; Rhei Radix et Rhizoma; } \\
\text { Chuanxiong Rhizoma }\end{array}$ \\
\hline Gao, $2017^{27}$ & Tang Shen Ning granule & $\begin{array}{l}\text { Astragali Radix; Euryales Semen; Rosae laevigatae Fructus; Rhei Radix et Rhizoma; } \\
\text { Chuanxiong Rhizoma }\end{array}$ \\
\hline Han, $2014^{28}$ & $\begin{array}{l}\text { Bao Shen pill; } \\
\text { Tripterygium glycosides }\end{array}$ & Not given \\
\hline Jia, $2015^{29}$ & $\begin{array}{l}\text { San Huang Yi Shen } \\
\text { granule }\end{array}$ & $\begin{array}{l}\text { Astragali Radix; Curcumae Longae Rhizoma; Rhei Radix et Rhizoma; Chuanxiong Rhizoma; } \\
\text { Angelicae Sinensis Radix; Salviae Miltiorrhizae Radix et Rhizoma; Cervi Cornu; Anemarrhenae } \\
\text { Rhizoma; Arctii Fructus }\end{array}$ \\
\hline $\begin{array}{l}\mathrm{Li}, 2012 \\
3146\end{array}$ & Modified Qi Wei granule & $\begin{array}{l}\text { Astragali Radix; Rehmanniae Radix; Prunellae Spica; Rhei Radix et Rhizoma; Euonymus Alatus; } \\
\text { Epimedii Folium; Corni Fructus; Curcumae Longae Rhizoma }\end{array}$ \\
\hline Lin, $2000^{32}$ & Tang Wei Kang capsule & stragali Radix; Ligustri Lucidi Fructus; Rhei Radix et Rhizoma \\
\hline
\end{tabular}

All ingredients are the standarised pharmaceutical name from the Chinese Pharmacopoeia 2015.

*Monascus purpureus Went. (Red Rice Yeast); pharmaceutical name not included in Chinese Pharmacopoeia 2015.

of eGFR than the actual eGFR and these positive results should be interpreted cautionsly.

One small study (44 participants) provided low quality of evidence that CHM made no difference to placebo in terms of eGFR after 3 months intervention (table 3). ${ }^{45}$ When comparing CHM to placebo plus ACEi/ARB, meta-analysis results indicated that no significant difference in eGFR over 1-3 months treatment (low quality evidence; table 3). ${ }^{26272944}$

Secondary outcomes

Meta-analysis results of secondary outcomes are summarised in online table S3. When compared with placebo, the pooled estimated effects for both fasting blood glucose (FBG) $2527323638-41$ and HbA1c 2427323638394145 did not show additional benefit of CHM in lowering blood glucose. Likewise, summarised effects from three studies showed no statistical differences between the CHM and placebo groups for systolic and diastolic blood pressure. ${ }^{24} 3945$ CHM resulted in lower levels of total cholesterol, $^{24} 32$ 36-41 triglycerides $^{24} 32$ 36-41 and LDL-C, ${ }^{24} 36-41$ although HDL-C levels ${ }^{243236-41}$ were not statistically significant compared with placebo. However, the results were limited by substantial heterogeneity and the reason was 


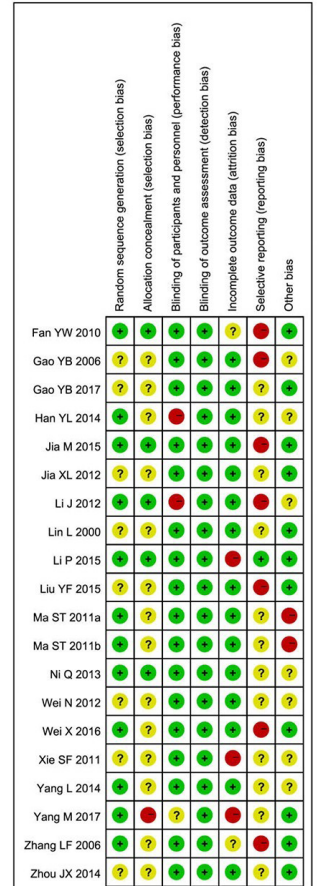

Figure 2 Risk-of-bias (ROB) of included studies Note: The red dot indicates high $\mathrm{ROB}$, yellow indicates unclear $\mathrm{ROB}$ and green dot indicates low ROB.

not found. Three studies ${ }^{24} 4246$ measured patients' QoL by questionnaire at the end of treatment but only two studies used the Diabetes QoL tool and provided usable data. The pooled estimation suggested no statistically significant differences between the CHM and the placebo group regarding QoL. ${ }^{24} 46$

\section{Safety evaluation of CHM therapy}

Data on adverse events was provided in 14 studies. Of these, seven studies stated no adverse events (AEs) were observed during study period. ${ }^{27} 293032333845$ In total, 53 cases of adverse events were reported in seven studies with 1445 participants. Except for Li's study, ${ }^{31}$ details of AEs in each group were reported. The most common $\mathrm{AE}$ of CHM was digestive system disorders (18 cases), including abdominal pain, diarrhoea or sloppy stools. ${ }^{343541}$ Both the CHM and control groups reported a modest number of cases of elevated liver enzyme levels (11 cases), infection (2 cases) or anaemia (3 cases). ${ }^{24} 2844$ In a three-arm study, ${ }^{44}$ one case of hypertension in the CHM group, one case of hypotension in losartan group and one case of hyperkalaemia in CHM plus losartan group were reported. All participants that experienced the AEs recovered after discontinuation of the tested interventions. Three cases of serious AEs, including two cases of death and a case of acute myocardial infarction (AMI), were reported in Li's trial. ${ }^{24}$ One participant in the CHM group died due to subarachnoid haemorrhage while another participant died after AMI. The researchers reported that these serious AEs were not related to the study agent.
Sensitivity and subgroup analysis

Sensitivity analysis by excluding studies with substantial ROB regarding randomisation showed consistent results with the primary analysis, except for the comparison of CHM versus placebo plus ACEi/ARB in terms of Scr level (online table S4). Subgroup analysis indicated that baseline kidney function, different CHM formulae and outcome measurement scales could partially explain the variant treatment effect of primary outcomes (online table S2). Publication bias was not evaluated due to the limited number of studies included in each outcome.

\section{DISCUSSION}

This review included 20 RCTs involving 2719 participants and evaluated the effects and safety of CHM in addition to conventional therapies for DKD. As an adjunctive therapy, CHM may decrease proteinuria (either measured as urinary albumin or protein excretion) in DKD patients compared with placebo, regardless of concomitant use of ACEi/ARB. When CHM and ACEi/ARB were used simultaneously, eGFR improved compared with ACEi/ ARB alone but studies had measurement shortfalls that may have overestimated the effect. CHM appeared to be well tolarated in DKD patients and no significant adverse events causal to CHM interventions were reported. These results suggest potential short-term renal benefit by adding CHM to conventional pharmacotherapies in DKD populations. However, due to the short follow-up periods and small numbers of clinical events in terms of mortality and progression to ESKD, the long-term benefit of CHM is yet to be determined.

This study demonstrated that CHM may be applied as an adjunctive treatment for DKD to achieve better renal outcomes. From the clinical perspective, the short-term albuminuria/proteinuria reduction effect of CHM identified in this review is moderate when compared with placebo. In patients with chronic kidney disease, the early reduction in albuminuria is associated with lower risk of ESKD or doubling Scr level, particulally in those patients with baseline albuminuria greater than $30 \mathrm{mg} / \mathrm{g} .{ }^{47}$ Therefore, for the subgroup of DKD patients who are contraindicated for ACEi/ARB use, CHM may offer some benefit. When used in combination with ACEi/ARB, the lowering albuminuria effect of CHM is mild to moderate from a clinical perspective. Considering the failure of dual RAS inhibitors therapy, CHM could be a potential option for those DKD patients who are on ACEi/ARB to achieve greater albuminuria reduction in the short-term. The combination of CHM and ACEi/ARB may also be benefitial in improving eGFR, especially for patients experiencing acute drop of eGFR after early RAS inhibitors initiation.

Findings from this review are in line with those of previous reviews focusing on single herbs or particular formulae. Li et al reviewed the clinical effect of preparations of Astragali Radix in DKD patients, finding that Astragali injection lowered Scr, increased eGFR and 


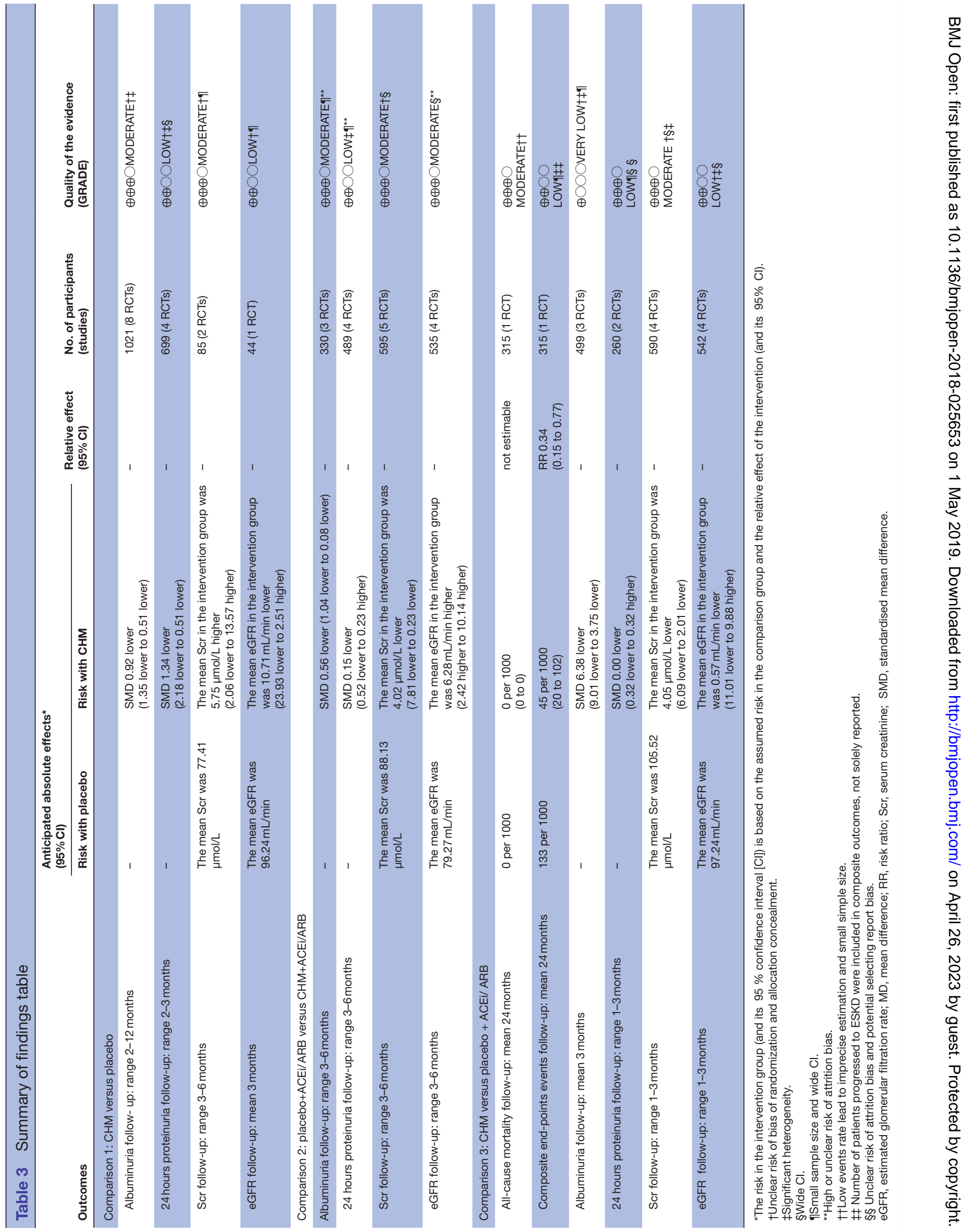




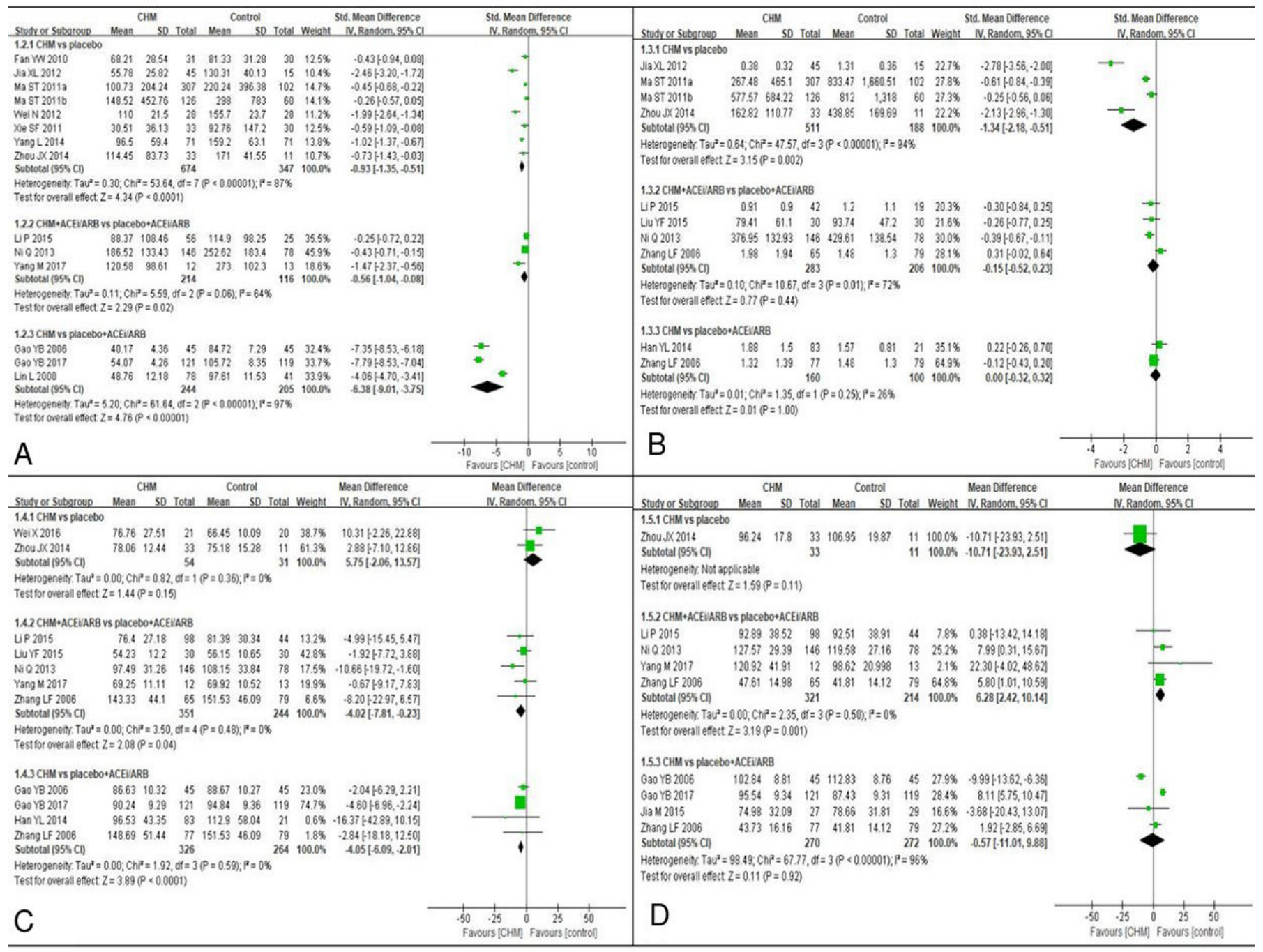

Figure 3 Forest plot of primary outcomes note: Panel (A) albuminuria outcomes; (B) proteinuria outcomes; (C) serum creatinine outcomes; (D) estimated glomerulus filtration rate outcomes. ACEi, angiotensin converting enzymeinhibitors; ARB, angiotensin receptor blockers; CHM, Chinese herbal medicine ; Std, standard.

reduced proteinuria based on data from 21 RCTs and four non-RCTs. ${ }^{48}$ In published reviews of Ginkgo Folium extract and Xue Zhi Kang capsules, lower fasting blood glucose and HbA1c levels in the CHM group were reported. ${ }^{49} 50$ The inconsistency in terms of the glycemic outcomes may have been due to differences in ingredients among the included studies. In our review, only two trials applied either Ginkgo Folium extract or Xue Zhi Kang capsules as interventions. The glycemic control effect may have been diluted by other trials using various herbal ingredients, which targeted on kidney rather than glycemic control. It should also be noted that the studies included in the previous reviews of Ginkgo Folium extract and Xue Zhi Kang capsules resulted in significant ROB (including publication bias). Thus, rigorous and large scale clinical trials are needed to confirm the glycemic control effects of CHM in DKD patients.

The renal protective effect of CHM may be related to particular bioactive compounds contained in the herbal ingredients included in these RCTs. The most frequently used herb was Astragali Radix. Both in vitro and in vivo studies have indicated that chemical components of Astragali Radix, such as Astragaloside IV and Astragalus saponin I, exert anti-oxidant and anti-inflammatory properties in diabetic models. ${ }^{51} 52$ These chemicals can prevent and restore kidney tissue injury related to oxidative stress. Additionally, Astragaloside IV can reduce endoplasmic reticulum stress and increase podocyte integrity, which is the therapeutic target for decreasing albuminuria. ${ }^{534}$ The second most frequently used herb, Rehmanniae Radix, also upregulates anti-inflammatory and antioxidant effects in diabetic rats. ${ }^{55}$ Furthermore, anti-diabetic properties were observed in its constituent compound (catalpol) and ethanolic extract. ${ }^{56}$ Although the glucose lowering effect of Rehmanniae Radix was not superior to metformin, its use was associated with higher anti-inflammatory activity, lower oxidative stress levels, and restoration of diabetes-induced kidney lesions. The third most frequent herb was Rhei Radix et Rhizoma. Active compounds of Rhei Radix et Rhizoma, 
including anthraquinones (rhein and emodin) and phenolic acids (gallic acid and ferulic acid), have been shown to protect the kidneys by reducing oxidative stress, inflammation, fibronectin and extracellular matrix accumulation. ${ }^{57-59}$ Furthermore, in vitro experiments have demonstrated that extracts of Rhei Radix et Rhizoma can inhibit lipid peroxidation and lower serum lipid levels, which are risk factors for diabetes and DKD progression. ${ }^{6061}$

Renal toxicity induced by aristolochic acid (AA) has been a concern since a series of renal failure cases caused by AA contaminated products were reported. ${ }^{62}{ }^{63}$ In our review, the CHM used in included studies appeared to be well-tolerated and safety signals were not identified. This could be related to the fact that all herbal ingredients investigated were free from $\mathrm{AA}$, and some of the studies mentioned a strict quality control processes regarding the CHM raw material and manufacturing procedures. ${ }^{24} 3144$ Mortality risk reduction effect of non-AA prescribed CHM was indicated in a chronic kidney disease population study, but for DKD patients, the long-term safety of CHM requires further studies to confirm. ${ }^{64}$

Although this review was conducted in a systematic and comprehensive manner, there are limitations that should be taken into account when interpreting the findings. First, the number of included studies was relatively small, and few studies measured and reported the same outcomes consistently. This caused difficulty in meta-analysis and introduced heterogeneity across studies and led to downgrading in quality of evidence. Even meta-analyses with low heterogeneity may not be reliable because there were only a very small number of included studies in the subgroup analyses (less than or equal to three studies in each subgroup). In addition, the positive effect of CHM in eGFR outcomes is dominated by a study using Cockcroft-Gault equation ( $64.8 \%$ weight), leading to possible overestimation of eGFR value.$^{65}$ Core outcome sets with standardised measurements are needed in future studies to rigorously assess the effect of CHM. Second, most of the studies had short follow-up periods (1-3 months) and small sample sizes, leading to imprecision of the estimated effect and low centainty with regard to long-term benefit and effect on renal function and clinical outcomes. Third, more than half of the included studies did not provide information on randomisation and allocation procedures, such that the impact of potential selection bias was unclear. Although the CHM formulae were processed as granules or capsules in order to achieve blinding, quality assurance information for each CHM preparation was not provided in most of the studies. Further studies are strongly encouraged to report following the CONSORT reporting guidelines with sufficient details regarding the manufacture and quality control of investigated CHM. ${ }^{66-68}$ Finally, although we did not limit the CHM interventions in terms of herbal composition, five included studies shared highly homologous CHM ingredients synthesis, ${ }^{24} 30314445$ thereby limiting the diversity of CHM treatments evaluated.
Since the participants in most included trials were older adults with a GFR greater than $60 \mathrm{~mL} / \mathrm{min}$, the renal protective effect of CHM in younger individuals and in advanced kidney disease is uncertain. Moreover, all included studies were conducted in China, such that the effect of CHM reported in this review may not be generalisable to other population groups. It should further be noted that, in most of the included studies, the forms of CHM used were multi-ingredients herbal formulae, which were developed based on traditional Chinese medicine theory and experts' clinical experience. While indicative from pharmacological studies, the most frequently used ingredients and formulae discussed above may not necessarily be relevant to the observed effects reported in this study.

\section{CONCLUSION}

In conclusion, combination of CHM with conventional RAS inhibitors showed promise as an adjunctive treatment for improving renal function and decreasing urinary albumin and protein excretion in patients with DKD. The rate of occurrences of adverse events was low and the tested CHM appeared to be well-tolerated. This systematic review also provided potential candidate formulae and frequently used herbs for further investigation. Well-designed RCTs following reporting guidelines with adequate sample sizes and longer follow-up periods are warranted to confirm the long-term efficacy and safety of CHM, especially with respect to patient-oriented outcomes such as mortality, disease progression and QoL.

\section{Author affiliations}

${ }^{1}$ Nephrology Department, the Second Affiliated Hospital of Guangzhou University of Chinese Medicine, Guangdong Provincial Hospital of Chinese Medicine, Guangdong Provincial Academy of Chinese Medical Sciences, Guangzhou, China

${ }^{2}$ The China-Australia International Research Centre for Chinese Medicine, RMIT University, Melbourne, Victoria, Australia

${ }^{3}$ Evidence-Based Medicine and Clinical Research Service Team, the Second Affiliated Hospital of Guangzhou University of Chinese Medicine, Guangdong Provincial Hospital of Chinese Medicine, Guangdong Provincial Academy of Chinese Medical Sciences, Guangzhou, China

${ }^{4}$ School of Health and Biomedical Sciences, RMIT University, Bundoora, Victoria, Australia

${ }^{5}$ Australia Kidney Trials Network, University of Queensland, Brisbane, Queensland, Australia

${ }^{6}$ Nephrology Department, Translational Research Institute, South Brisbane, Queensland, Australia

${ }^{7}$ Guangdong Provincial Key Laboratory of Clinical Research on Traditional Chinese Medicine Syndrome, Guangdong Provincial Academy of Chinese Medical Sciences, Guangzhou, China

Acknowledgements LaZ is supported by a Chinese Medicine Collaborative Research Training Scholarship from the China- Australia International Research Centre for Chinese Medicine (CAIRCCM). DJ is supported by an Australian National Health and Medical Research Council Practitioner Fellowship.

Contributors Research idea and study design: LaZ, CL, CCX and WM; data collection and screening: LaZ, LY, JS, XQ and ALZ; data extraction: LaZ, LY and JS; data analysis: LaZ and JS; risk of bias assessment: LaZ, LY, XG and ALZ; GRADE assessment: WM, LeiZ, LY, JS and DJ; manuscript writing: all authors; supervision and mentorship: CCX, XL and CL. Each author contributed important intellectual content during manuscript drafting and revision and accepts accountability for the 
overall work by ensuring that questions pertaining to the accuracy or integrity of any portion of the work are appropriately investigated and resolved.

Funding The project is jointly supported by the China-Australia International Research Centre for Chinese Medicine (CAIRCCM)—a joint initiative of RMIT University, Australia and the Guangdong Provincial Academy of Chinese Medical Sciences, China with additional funding support from the Ministry of Science \& Technology of China (International Cooperation Project, Grant Number 2012DFA31760) and a grant from the National Natural Science Foundation of China (Grant Number 81603717).

Competing interests None declared.

Patient consent for publication Not required.

Provenance and peer review Not commissioned; externally peer reviewed.

Data sharing statement Data extracted from original studies and data used for meta-analysis are available on request.

Open access This is an open access article distributed in accordance with the Creative Commons Attribution Non Commercial (CC BY-NC 4.0) license, which permits others to distribute, remix, adapt, build upon this work non-commercially, and license their derivative works on different terms, provided the original work is properly cited, appropriate credit is given, any changes made indicated, and the use is non-commercial. See: http://creativecommons.org/licenses/by-nc/4.0/.

\section{REFERENCES}

1. White SL, Chadban S, Report K. Kidneys in Diabetes): temporal trends in the epidemiology of diabetic kidney disease and the associated health care burden in Australia. Report of the Kidney in Diabetes. Melbourne, Australia: Kidney Health Australia, 2014.

2. Saran R, Li Y, Robinson B, et al. US renal data system 2014 annual data report: epidemiology of kidney disease in the United States. Am $J$ Kidney Dis 2015;66:A7-305.

3. Sarnak MJ, Levey AS, Schoolwerth AC, et al. Kidney disease as a risk factor for development of cardiovascular disease: a statement from the American Heart Association Councils on Kidney in Cardiovascular Disease, High Blood Pressure Research, Clinical Cardiology, and Epidemiology and Prevention. Circulation 2003;108:2154-69.

4. National Kidney Foundation. KDOQI clinical practice guidelines and clinical practice recommendations for diabetes and chronic kidney disease. Am J Kidney Dis 2007;49:S12-154.

5. National Kidney Foundation. KDOQI clinical practice guideline for diabetes and CKD: 2012 update. Am J Kidney Dis 2012;60:850-86.

6. Kramer H, Boucher RE, Leehey D, et al. Increasing mortality in adults with diabetes and low estimated glomerular filtration rate in the absence of albuminuria. Diabetes Care 2018;41:775-81.

7. Krolewski AS, Skupien J, Rossing P, et al. Fast renal decline to end-stage renal disease: an unrecognized feature of nephropathy in diabetes. Kidney Int 2017;91:1300-11.

8. Afkarian M, Zelnick LR, Hall YN, et al. Clinical Manifestations of Kidney Disease Among US Adults With Diabetes, 1988-2014. JAMA 2016;316:602-10.

9. Muskiet MHA, Wheeler DC, Heerspink HJL. New pharmacological strategies for protecting kidney function in type 2 diabetes. Lancet Diabetes Endocrinol 2018

10. de Zeeuw D, Akizawa T, Audhya P, et al. Bardoxolone methyl in type 2 diabetes and stage 4 chronic kidney disease. N Engl J Med 2013;369:2492-503.

11. Packham DK, Wolfe R, Reutens AT, et al. Sulodexide fails to demonstrate renoprotection in overt type 2 diabetic nephropathy. $J$ Am Soc Nephrol 2012;23:123-30.

12. Zhang L, Li Y, Guo X, et al. Text mining of the classical medical literature for medicines that show potential in diabetic nephropathy. Evid Based Complement Alternat Med 2014;2014:1-12.

13. China Academy of Chinese Medical Sciences. Evidence-based guidelines of clinical practice in Chinese medicine-internal medicine. Beijing: China Press of Traditional Chinese Medicine, 2011. 中国中医 科学院. 中医循证临床实践指南: 中医内科分册. 北京: 中国中医药出版 社.

14. Gao YB, Liu TH, Nan Z, et al. The Chinese medicine diagnosis and treatment standards of diabetic nephropathy. World Journal of Integrated Traditional and Western Medicine 2011;6:548-52. 糖尿病 肾脏疾病中医诊疗标准. 世界中西医结合杂志.

15. Lord GM, Cook T, Arlt VM, et al. Urothelial malignant disease and Chinese herbal nephropathy. Lancet 2001;358:1515-6.

16. Stanifer JW, Kilonzo K, Wang D, et al. Traditional medicines and kidney disease in low- and middle-income countries: opportunities and challenges. Semin Nephrol 2017;37:245-59.
17. Higgins JPT, Green S. Cochrane handbook for systematic reviews of interventions (Version 5.1.0). The Cochrane Collaboration 2011.

18. Moher D, Liberati A, Tetzlaff J, et al. Preferred reporting items for systematic reviews and meta-analyses: the PRISMA statement. Ann Intern Med 2009;151:264-9.

19. Mogensen CE, Christensen CK, Vittinghus E. The stages in diabetic renal disease. With emphasis on the stage of incipient diabetic nephropathy. Diabetes 1983;32:64-78.

20. Yang NZ, Diagnosis LXS. syndrome diffeciation and treatment effect eveluation of diabetic nephropathy (protocal). Shanghai Journal of Traditional Chinese Medicine 2007;41:7-8. 杨霓芝, 刘旭 生. 糖尿病肾病诊断, 辨证分型及疗效评定标准 (试行方案). 上海中医 药杂志

21. The Nordic Cochrane Centre, The Cochrane Collaboration. Review Manager (RevMan). Copenhagen, Denmark: The Nordic Cochrane Centre, The Cochrane Collaboration, 2014.

22. Higgins JP, Altman DG, Gøtzsche PC, et al. The Cochrane Collaboration's tool for assessing risk of bias in randomised trials. BMJ 2011;343:d5928.

23. Schünemann H, Brozek J, Guyatt G. GRADE handbook for grading quality of evidence and strength of recommendation. https://gdt. gradepro.org/app/handbook/handbook.html (Accessed 19 Oct 2017).

24. Li P, Chen Y, Liu J, et al. Efficacy and safety of tangshen formula on patients with type 2 diabetic kidney disease: a multicenter double-blinded randomized placebo-controlled trial. PLoS One 2015;10:e0126027.

25. Fan YW. Clinical study of Qi Kwai granules in early type 2 diabetic nephropathy. Naijing: Nanjing University of Chinese Medicine, 2010. 范译文. 芪葵颗粒干预早期 2 型糖尿病肾病的临床研究. 南京中医药大 学, 硕士论文.

26. Gao YB, Zhao HL, Guan S, et al. Clinical research of Tang Shen Ning in treating early stage of diabetic nephropathy with types of deficiency of both vital energy and yin and collaterals siltation and stagnant. China Journal of Traditional Chinese Medicine and Pharmacy 2006:409-11. 糖肾宁治疗气阴两虚、络脉疼滞型早期糖尿 病肾病临床研究.

27. Gao YB, Zhou HL, Guan S, et al. Multicenter randomized and double-blind controlled clinical trial of Tang Shen Ning Granules in treating diabetic kidney disease. Chinese Journal of Traditional Chinese Medicine 2017;32:5212-5. 糖肾宁颗粒治疗糖尿病肾病多中 心随机双盲对照临床试验.

28. Han YL. Method of combination of disease and syndrome in treating stage IV proteinuria in diabetic nephropathy of Qi-Yin deficiency. Journal of Changchun University of Traditional Chinese Medicine 2014;30:903-5. 病证结合法治疗气阴两虚型糖尿病肾病IV期蛋白尿.

29. Jia M. Clinical research of Sanhuang Yishen granule on patients diabetic nephropathy in IV stage. World Chinese Medicine 2015;10. 三黄益肾颗粒干预糖尿病肾病IV期的临床研究.

30. Jia XL. Study on clinic effect and experiment research of Qiwei treating the DN. Beijing: Beijing University of Chinese Medicine, 2012. 贾晓蕾. 芪卫颗粒干预糖尿病肾病临床及实验研究. 北京中医药大 学, 硕士论.

31. Li J, Zhao JX, Wang SD, et al. Effect of the whole course intervention program of combined therapy of TCM on the endpoint events of diabetic nephropathy. Journal of Traditional Chinese Medicine 2012;53:568-71. 中医药综合治疗方案全程干预对糖尿病肾病终点事件 的影响.

32. Lin L, Ni Q, Gao Q, et al. [Clinical study on tangweikang capsule in treating diabetic nephropathy]. Zhongguo Zhong Xi Yi Jie He Za Zhi 2000;20:811-4. 糖微康胶囊治疗糖尿病肾病的临床观察.

33. Liu YF. Qihuang capsule in treatment of diabetic nephropathy Qi and Yin deficiency syndrome clinical. Harbin: Heilongjiang University Of Chinese Medicine, 2015. 芪黄胶囊治疗糖尿病肾病气阴两虚型的临床 观察.

34. St M, Liu DL, Niu R, et al. Double -blind randomized placebocontrolled multi-centre phase III clinical trial of Arctiin granule in the treatment of diabetic nephropathy. Chin J Clin Pharmacol 2011;27. 牛蒡子苷治疗糖尿病肾病的随机双盲安慰剂多中心川I期临床试验.

35. St M, Liu DL, Niu R, et al. Tangjiangshekang granule in treatment of diabetic nephropathy: a double -blind, randomized, placebocontrolled multicentre clinical trial. Chin J New Drugs Clin Rem 2011;30:16-19. 糖降肾康颗粒治疗糖尿病肾病随机双盲安慰剂对照多 中心临床试验.

36. Ni Q, Jiang S, Xiao YX, et al. A clinical observation on Qiyao Xiaoke capsule combined with western medicine for 146 cases of Diabetic Nephropathy. Journal of Traditional Chinese Medicine 2013;54:484-7. 芪药消渴胶囊联合西药治疗糖尿病肾病146例临床观察.

37. Wei N, Chang WS, Xue DZ, et al. Effect of Xuezhikang on oxidative stress in early diabetic nephropathy. Chinese General Practice 2012;15(18:2085-7. 血脂康对早期糖尿病肾病患者氧化应激的影响. 
38. Wei X, Yao HJ, Liu Y, et al. Efficacy observation of adjunctive treatment of gandi capsules for diabetic nephropathy. China Pharmacy 2016;27:225-7. 甘地胶囊辅助治疗糖尿病肾病的疗效观察.

39. Xie SF, Huang LJ, Liu JS, et al. The long-term effect of Yang yin he luo medicinals for reducing urinary albumin to creatinine ratio in early diabetic nephropathy patients. Jiangsu $J$ Tradit Chin Med 2011;43:19-20. 长期应用养阴和络中药对早期糖尿病肾病患者尿微量 白蛋白肌酐比值的影响.

40. Yang L, Tan ZH, YK L, et al. The clinical effect observation of Qiming granules with standard care for early diabetic nephropathy. Guiding Journal of Traditional Chinese Medicine and Pharmacy 2014;20:52-3. 芪明颗粒配合常规疗法治疗早期糖尿病肾病71例临床观 察.

41. Yang M. Study on the clinical exploratory study of Qizhu granule in the treatment of early type 2 diabetic nephropathy. Beijing: China Academy of Chinese Medical Sciences, 2017. 杨明. 芪术颗粒治疗早 期2型糖尿病肾病临床探索性试验研究. 中国中医科学院, 硕士论文.

42. Zhang LF. Effect of TCM treatment on quality of life in diabetic nephropathy patients with renal failure: a multi-centered clinical study. Journal of Traditional Chinese Medicine 2008;49:119-22. 中医 辨证治疗方案对糖尿病肾病肾功能不全患者生存质量的影响-多中心临 床研究.

43. Zhang LF, Zhao JX, Rh L, et al. The effect of Differiation Chinese Medicine Treatment on Syndrome Improvement in diabetic nephropathy patients with renal failure. Abstract of the forth International Integration Medicine Conference of Kidney Diseases. Tian jin, China, 2006. 中医辨证论治糖尿病肾病肾功能不全证候疗效评 价研究. 第四届国际中西医结合肾脏病学术会议.

44. Zhang LF, Zhao JX, Rh L, et al. Study on the effectiveness and safety of optimum program of prevention and treatment of renal insufficiency of diabetic nephropathy. China Foreign Medical Treatment 2006;47:755-8. 糖尿病肾病肾功能不全防治优化方案的有效 性和安全性研究.

45. Zhou JX. Effect and safety of Qiwei granule on diabetic nephropathy and podocyte. Beijing: Beijing University of Chinese medicine, 2014. 周静金菳. 芪卫颗粒干预糖尿病肾病临床疗效及其保护足细胞作用机制研 究. 北京中医药大学, 硕士论文.

46. Zhou X, Zhao JX, Wang SD, et al. Effect of TCM Comprehensive Treatment Program on Quality of Life in Patients With Diabetic Nephropathy. Journal of Traditional Chinese Medicine 2014;55:473-7. 中医药综合治疗方案对糖尿病肾病患者生存质量的影响.

47. Heerspink HJL, Greene T, Tighiouart $\mathrm{H}$, et al. Change in albuminuria as a surrogate endpoint for progression of kidney disease: a metaanalysis of treatment effects in randomised clinical trials. Lancet Diabetes Endocrinol 2019;7:128-39.

48. Li M, Wang W, Xue J, et al. Meta-analysis of the clinical value of Astragalus membranaceus in diabetic nephropathy. $J$ Ethnopharmacol 2011;133:412-9.

49. Wang F, Hm W. Xuezhikang for diabetic kidney disease: a systematic review of randomized controlled trials. Chin J Evid-based Med 2009;9:63-70. 血脂康治疗糖尿病肾病的系统评价.

50. Zhang L, Mao W, Guo X, et al. Ginkgo biloba extract for patients with early diabetic nephropathy: a systematic review. Evid Based Complement Alternat Med 2013;2013:689142.

51. Gui D, Huang J, Guo Y, et al. Astragaloside IV ameliorates renal injury in streptozotocin-induced diabetic rats through inhibiting NF- $\mathrm{KB}-$ mediated inflammatory genes expression. Cytokine 2013;61:970-7.

52. Qi W, Niu J, Qin Q, et al. Astragaloside IV attenuates glycated albumin-induced epithelial-to-mesenchymal transition by inhibiting oxidative stress in renal proximal tubular cells. Cell Stress Chaperones 2014;19:105-14.

53. Gui D, Guo Y, Wang F, et al. Astragaloside IV, a novel antioxidant, prevents glucose-induced podocyte apoptosis in vitro and in vivo. PLoS One 2012;7:e39824.

54. Wang ZS, Xiong F, Xie XH, et al. Astragaloside IV attenuates proteinuria in streptozotocin-induced diabetic nephropathy via the inhibition of endoplasmic reticulum stress. BMC Nephrol 2015;16:44.

55. Waisundara VY, Huang M, Hsu A, et al. Characterization of the anti-diabetic and antioxidant effects of rehmannia glutinosa in streptozotocin-induced diabetic Wistar rats. Am J Chin Med 2008;36:1083-104

56. Yang S, Deng H, Zhang Q, et al. Amelioration of diabetic mouse nephropathy by catalpol correlates with down-regulation of Grb10 expression and activation of insulin-like growth factor 1 / insulin-like growth factor 1 receptor signaling. PLoS One 2016;11:e0151857.

57. Zhang ZH, Wei F, Vaziri ND, et al. Metabolomics insights into chronic kidney disease and modulatory effect of rhubarb against tubulointerstitial fibrosis. Sci Rep 2015;5:14472.

58. Ahad A, Ahsan H, Mujeeb M, et al. Gallic acid ameliorates renal functions by inhibiting the activation of p38 MAPK in experimentally induced type 2 diabetic rats and cultured rat proximal tubular epithelial cells. Chem Biol Interact 2015;240:292-303.

59. Zeng CC, Liu X, Chen GR, et al. The molecular mechanism of rhein in diabetic nephropathy. Evid Based Complement Alternat Med 2014;2014:1-6.

60. Hosseini A, Mollazadeh H, Amiri MS, et al. Effects of a standardized extract of Rheum turkestanicum Janischew root on diabetic changes in the kidney, liver and heart of streptozotocin-induced diabetic rats. Biomed Pharmacother 2017;86:605-11.

61. Punithavathi VR, Prince PS, Kumar R, et al. Antihyperglycaemic, antilipid peroxidative and antioxidant effects of gallic acid on streptozotocin induced diabetic Wistar rats. Eur J Pharmacol 2011;650:465-71.

62. Vanherweghem JL, Depierreux M, Tielemans C, et al. Rapidly progressive interstitial renal fibrosis in young women: association with slimming regimen including Chinese herbs. Lancet 1993;341:387-91.

63. Nortier JL, Martinez M-CM, Schmeiser HH, et al. Urothelial Carcinoma Associated with the Use of a Chinese Herb (Aristolochia fangchi). N Engl J Med Overseas Ed 2000;342:1686-92.

64. Hsieh CF, Huang SL, Chen CL, et al. Non-aristolochic acid prescribed Chinese herbal medicines and the risk of mortality in patients with chronic kidney disease: results from a population-based follow-up study. BMJ Open 2014;4:e004033.

65. Levey AS, Stevens LA. Estimating GFR using the CKD Epidemiology Collaboration (CKD-EPI) creatinine equation: more accurate GFR estimates, lower CKD prevalence estimates, and better risk predictions. Am J Kidney Dis 2010;55:622-7.

66. Gagnier J, Boon H, Rochon P, et al. Improving the quality of reporting of randomized controlled trials evaluating herbal interventions: implementing the CONSORT statement [corrected]. Explore 2006;2:143-9.

67. Gagnier JJ, Boon H, Rochon P, et al. Reporting randomized, controlled trials of herbal interventions: an elaborated CONSORT statement. Ann Intern Med 2006;144:364-7.

68. Schulz KF, Altman DG, Moher D, et al. CONSORT 2010 statement: updated guidelines for reporting parallel group randomised trials. BMJ 2010;340:c332. 